\title{
Computation in Undergraduate Physics: The Lawrence Approach
}

\author{
David M. Cook \\ Department of Physics \\ Lawrence University \\ Appleton, WI 54912 \\ USA \\ david.m.cook@lawrence.edu
}

\begin{abstract}
Most efforts using computers in physics curricula focus on introductory courses or individual upper-level courses. In contrast, for a dozen years the Lawrence Department of Physics has been striving to embed the use of general purpose graphical, symbolic, and numeric computational tools throughout our curriculum. Developed with support from the (US) National Science Foundation, the Keck Foundation, and Lawrence University, our approach involves introducing freshman to tools for data acquisition and analysis, offering sophomores a course that introduces them to symbolic, numerical, and visualization tools, incorporating computational approaches alongside traditional approaches to problems in many intermediate and upper level courses, and making computational resources available so that students come to see them as tools to be used routinely on their own initiative whenever their use seems appropriate. A text reflecting the developments at Lawrence is in preparation, will undergo beta testing in 2001-02, and will be published in January, 2003. Details about the Lawrence curricular approach and the emerging text can be found from links at www.lawrence.edu/dept/physics.
\end{abstract}

For a dozen years or more (and with support from the National Science Foundation [1, the W. M. Keck Foundation 2], and Lawrence University), we in the Department of Physics at Lawrence University 11 have been developing the computational dimensions of our upper-level curriculum 3/4,56]. We have built a computational laboratory that makes a wide spectrum of hardware and software available to students, developed an approach that introduces students to these resources and to prototypical applications, and drafted several hundred pages of instructional materials that, with support from a recent (US) National Science Foundation (NSF) grant [7, are currently being prepared for publication. This paper

1. lays out the underlying convictions that have guided the development of our approach to incorporating computation in an undergraduate curriculum,

\footnotetext{
${ }^{1}$ Lawrence University is a liberal arts college and conservatory of music with about 1250 students. The Department of Physics has five full time members and graduates an average of a dozen majors each year.
}

V.N. Alexandrov et al. (Eds.): ICCS 2001, LNCS 2073, pp. 1074-1083 2001.

(C) Springer-Verlag Berlin Heidelberg 2001 
2. describes the Lawrence curricular components,

3. discusses particularly the sophomore course that is the starting point for those students who pursue computation most aggressively, and

4. describes the instructional materials currently being prepared for publication.

\section{Underlying Convictions}

The primary tasks of those parts of an undergraduate physics program that focus on physics majors are to awaken in our students a full realization of the beauty, breadth, and power of the discipline and to help them develop both a secure understanding of fundamental concepts and the skills to use a variety of tools in applying those concepts. Among the tools, we at Lawrence would firmly include computational resources of several sorts. We believe

1. that our curricula must familiarize students

a) with the functions and capabilities of at least one operating system.

b) with the use of at least one good text editor (not word processor).

c) with several types of computational tool, including

i. a spreadsheet like Excel[8].

ii. resources like IDL[9] and MATLAB[10] for numerical processing of numbers and arrays.

iii. C and FORTRAN programming sufficient to permit comfortable use of subroutine packages like Numerical Recipes[11] and LSODE[12].

iv. resources like MAPLE[13] and MATHEMATICA[14] for symbolic manipulation of expressions.

v. resources like Kaleidagraph[15], IDL, MATLAB, and IRIS/NAG Explorer[16] for graphical visualization of complex data.

vi. resources like $\mathrm{AT}_{\mathrm{E}} \mathrm{X}[17]$ and tgif [18] for preparing technical reports and manuscripts.

d) with several types of symbolic and numerical analyses, including solving algebraic equations, solving ordinary and partial differential equations, evaluating integrals, finding roots, performing data analyses, fitting curves to experimental data, and manipulating images.

e) with the assessment of accuracy in finite-precision arithmetic.

2. that students must be introduced early to these tools. An upper-level course in computational physics is a valuable curricular inclusion, but students need to become acquainted with computational resources long before they have either the mathematical or the physical background to profit from a rigorous computational physics course.

3. that use of computational resources must permeate the curriculum.

4. that the initial encounter with computational tools cannot be effectively accomplished as an appendix to tasks given higher priority. Certainly, numerous examples drawn from physical contexts must be used to motivate study of techniques and tools, but the focus must be on the features and capabilities of the tools. 
Table 1. The typical program of a Lawrence physics major. Courses shown in bold type have explicit computer content.
Term I
Term II
Term III
Year 1: Social Science Elective Intro Classical Physics Intro Modern Physics Calculus I $\quad$ Calculus II Calculus III
Freshman Studies Freshman Studies Free Elective

$\begin{array}{cll}\text { Year 2: } \text { Electronics } & \text { Mechanics } & \mathbf{E} \text { and } \mathbf{M} \\ \text { Linear Algebra/ODE } & \text { Humanities Elective } & \text { Humanities Elective } \\ \text { Free Elective } & \text { Free Elective } & \text { Free Elective }\end{array}$

Year 3: Quantum Mechanics Advanced Laboratory Physics Elective Language Language Language Social Science Elective Free Elective Free Elective

$\begin{array}{cll}\text { Year 4: Free Elective* } & \text { Physics Elective } & \text { Physics Elective } \\ \text { Diversity Elective } & \text { Free Elective } & \text { Diversity Elective } \\ \text { Free Elective } & \text { Free Elective } & \text { Free Elective }\end{array}$

* Often independent research in physics.

In the broadest of terms, we should be structuring our curricula so that, ultimately, students will recognize when a computational approach may have merit and will be prepared to pursue that approach confidently, fluently, effectively, knowledgeably, and independently whenever they deem it appropriate.

The Lawrence approach to nurturing the abilities of students to use computational resources is active; it compels students to play a personal role in their own learning; it forces students to defend their work in writing; it gives students practice in preparing and delivering oral presentations; it encourages students to work in groups; it permeates our curriculum; and, more than any other objective, it develops the students' abilities to operate in this arena on their own initiative.

\section{The Curricular Context}

An efficient way to describe the Lawrence approach is to track the computational experience of an entering freshman physics major as she moves towards graduation four years later. Each year, full-time students at Lawrence take three courses in each of three ten-week terms. Class periods are 70 minutes long, and a one-term course translates officially into $3-1 / 3$ semester hours. While there are many variations, the typical program of a student pursuing a physics major is shown in Table 1. This table also shows the area-though not necessarily the actual term - of courses needed to satisfy general education requirements. The minimum physics major is satisfied by ten courses in physics, seven of which are 
Table 2. Available Electives. Again, courses shown in bold type have explicit computer content.

- Thermal Physics

- Optics

- Solid State Physics

- Advanced Modern Physics

- Laser Physics
- Advanced E and M

- Math Methods

- Advanced Mechanics

- Plasma Physics

- Tutorial in Physics

- Computational Tools in Physics

- Independent Study in Physics

explicitly stipulated, and four courses in mathematics. Courses shown in bold type direct students explicitly to the computer and, in most cases, include some instruction in one or more of our computational resources.

Available physics electives are shown in Table 2 Again, entries in bold type make explicit use of computational resources. In the other courses, students use those resources regularly on their own initiative. Majors are required to take three courses from the top group of nine and may take as many as five more from the entire spectrum before exceeding an institutionally imposed limit of 15 courses in any single department. Tutorials and independent studies, the latter being elected by nearly all senior majors and sometimes extending over more than one term and leading to honors in independent study at graduation, offer a vehicle for students to study topics not included in our regular course offerings.

\section{The Computational Components}

Prospective physics majors at Lawrence first encounter computational approaches in the introductory courses, whose laboratory is equipped with Macintosh computers, Vernier ULI cards[19, and a variety of sensors. Beyond LoggerPro, students have access both in the laboratory and elsewhere on campus to Excel and Kaleidagraph. Exercises assigned in the laboratory routinely involve automated data acquisition, statistical analysis, and curve fitting; exercises assigned in lectures occasionally send students to the laboratory computers for graphing results or pursuing numerical solutions to Newton's laws with editable Excel templates. By the end of the freshman year, prospective majors have already developed some skills in the use of computational tools, particularly skills of value in the laboratory.

Beyond the freshman year, students - of course - continue to use Excel and Kaleidagraph, but they also have access to our Computational Physics Laboratory (the CPL), which is equipped with six Silicon Graphics UNIX workstations, monochrome and color printers, and software in all the categories enumerated above. Each student has an account in this departmental facility, and each is entitled to a key both to the CPL and to the building, so each has 24/7 access to the CPL. 
To help sophomores become confident, regular users of the CPL, we offer a course called Computational Tools in Physics, to be described in the next section. Even those sophomores who don't elect this course, however, encounter two short computational workshops - one on IDL and the other on MAPLE - in our required sophomore mechanics course. Thus, all sophomores have at least a small, forced exposure to the CPL, and some - but unfortunately not all-sophomores have a fully comprehensive introduction to the available capabilities. Subsequent theoretical and experimental courses alike offer students many opportunities to continue honing their computational skills and, depending on the instructor, some of these courses will direct students explicitly to the CPL for an occasional exercise. Most senior capstone projects will use the resources of the CPL, at least for visualization of data and/or preparation of reports. Some projects, notably those in fluid mechanics, musical acoustics, xray diffraction, mapping of astrophysical data, and multiphoton quantum transitions, have made extensive use of these facilities. Some physics students use the CPL in conjunction with courses in other departments, particularly mathematics.

\section{The Sophomore Course}

An elective course called Computational Tools in Physics is the starting point in our nurturing of our students' abilities to take full advantage of the resources of the CPL. Currently, this full-credit course is offered in three 1/3-credit segments, one in each of the three terms of our academic year. Its topics are coordinated with the required courses taken by sophomore majors. The first term focuses on acquainting students with the rudimentary capabilities of our CPL. It starts with a tutorial orientation to UNIX (1 week) and then addresses array processing and graphical visualization using IDL (2 weeks), publishing scientific manuscripts using $\mathrm{IAT}_{\mathrm{E}} \mathrm{X}$ and tgif (1 week), graphical visualization using IRIS/NAG Explorer ( 2 weeks), symbolic manipulations using MAPLE (2 weeks), and circuit simulation using SPICE[20 (2 weeks). In each class, students are introduced to a particular computational tool. Then, each student works several exercises, ultimately turning in written solutions prepared with $\mathrm{LT}_{\mathrm{E}} \mathrm{X}$. The half-dozen class sessions in the term provide only orientation and motivation; students are expected to exhibit a fair bit of personal independence and aggressiveness as they progress from the starting point provided by the classes to the knowledge and skill needed to finish the assignments.

The second term is coordinated with an intermediate course in classical mechanics, for which Barger and Olsson 21] is the current text, and focuses on symbolic and numerical approaches to ordinary differential equations (ODEs). In the first half of the term, the course covers symbolic solution of ODEs and Laplace Transforms with MAPLE (2 weeks), numerical solution of ODEs with IDL (2 weeks), and numerical solution of ODEs with FORTRAN programs and LSODE (1 week). Each student completes this term by carrying out an extended project that culminates in a written paper and a 20 -minute oral presentation to 
the class. Topics like the three-body problem, coupled oscillators, the compound pendulum, anharmonic oscillators, and chaos have been addressed.

The third term is coordinated with an intermediate course in electricity and magnetism, for which Griffiths 22 is the current text, and focuses on symbolic and numerical integration. In the first six weeks of the term, the course covers symbolic and numerical integration with MAPLE and IDL (2 weeks), numerical integration using FORTRAN programs and Numerical Recipes (2 weeks), and root finding using MAPLE, IDL, and Numerical Recipes (2 weeks). This term also concludes with an extended project, written paper, and 20-minute oral presentation. Topics like electric fields and potentials, magnetic fields, Fourier analysis, non-linear least squares fitting, and global positioning systems have been addressed.

\section{The Emerging Text}

The NSF grant 7] already mentioned provides support for converting the experience acquired and the library of instructional materials developed at Lawrence into a flexible publication 23 as a resource for other institutions. That we don't all use the same spectrum of hardware and software, however, poses a major challenge. The variety of options and combinations is so great that any single choice (or coordinated set of choices) is bound to limit the usefulness of the end result to a small subset of all potentially interested users. The strategy adopted to address that challenge involves assembling different incarnations of the basic materials from a wide assortment of components, some of which - the generic components - will be included in all incarnations and others of which - those specific to particular software packages - will be included only if the potential user requests them. Thus, the specific software and hardware treated in any particular incarnation will be microscopically "tailor-able" to the spectrum of resources available at the instructor's site. One incarnation, for example, could include the generic components and only the components that discuss IDL, MAPLE, C, and $\mathrm{IT}_{\mathrm{E}} \mathrm{X}$ while another might include the generic components and the components that focus on MATLAB, MATHEMATICA, and FORTRAN (including Numerical Recipes).

While the materials are still very much being refined, the present tentative table of contents includes the chapters and appendices listed in Table 3, In this structure,

1. Chapter 1 stands alone; chapters $2-5$ introduce the general features of an array processor, a computer algebra system, a programming language, and the numerical recipes library; Chapters 6-12 address several important categories of computational processing; and the appendices introduce the use of an operating system, a publishing system, and a program for producing drawings.

2. In addition to the options explicitly indicated, later chapters also contain internal options that are not shown. 
Table 3. Tentative table of contents for the book tentatively titled Computation and Problem Solving in Undergraduate Physics.

1. Overview

2. Introduction to IDL and/or MATLAB and/or ...

3. Introduction to MACSYMA and/or MAPLE and/or Mathematica and/or ...

4. Introduction to Programming in FORTRAN and/or $\mathrm{C}$ and/or ...

5. Introduction to Numerical Recipes

6. Solving Ordinary Differential Equations

7. Introduction to LSODE

8. Evaluating Integrals

9. Finding Roots

10. Solving Partial Differential Equations

11. Data Analysis/Curve Fitting

12. Fourier Analysis and Image Processing

A. Introduction to UNIX and/or Windows and/or ...

B. Introduction to $\mathrm{AT}_{\mathrm{E}} \mathrm{X}$ and/or Word and/or ...

C. Introduction to TGIF and/or ...

3. The order of presentation in the book does not compel any particular order of treatment in a course or program of self-study. While some later sections depend on some earlier sections, the linkages are not particularly tight.

While the objective is for students to become fluent in the use of a spectrum of computational tools - and the chapters are organized by program or by computational technique, the motivation throughout lies in and all examples are drawn from physical contexts.

Chapter 2, the tentative table of contents for the MATLAB version of which is shown in Table 4, represents chapters that introduce basic features of an application program, specifically a program for processing arrays of numbers and creating graphical visualizations of one-, two-, and three-dimensional data sets. The bulk of the chapters in this category are structured as tutorials and lean in some measure on vendor-supplied documentation and on-line help to encourage and guide self-study.

Shown in Table 5 the structure of Chapter 8 on evaluating integrals exemplifies that of all of the chapters on various computational techniques. Presumably, before approaching any particular section in this chapter, the student would have studied the relevant sections in earlier chapters. The first section, whose detail is laid out in the next paragraph, sets several physical problems, the successful addressing of which benefits from exploitation of a computational tool. The second section describes how one might use a symbolic tool in application to some of the problems set in the first section. Save for the last, the remaining sections describe suitable numerical algorithms generically and then illustrate how those algorithms can be invoked in a variety of ways. The final section lays out several exercises that students can use to hone their skills. Sections 8.1, 8.3, and 8.7 would be included in all versions of the chapter; each individual instructor 
Table 4. Sections in the chapter on MATLAB. The IDL chapter is similar.

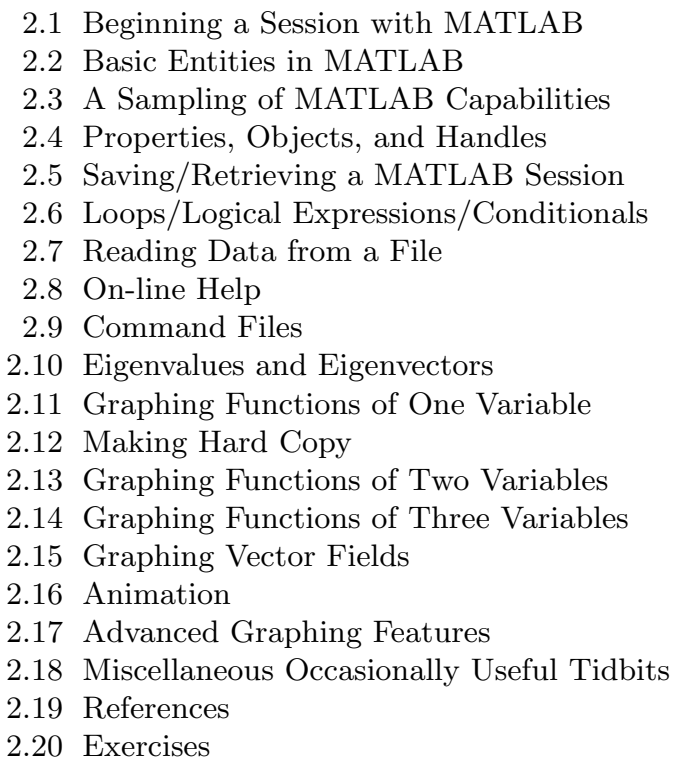

would select from the indicated options only those that are appropriate to that instructor's site.

Yet one level further down in the envisioned structure, Table 6] shows the present list of sample problems for Chapter 8. They range over several subareas of physics and reveal that evaluation of integrals, perhaps as functions of one or more parameters, plays an important role in many areas of physics.

Even among sites that use the same spectrum of hardware and software, however, some aspects of local environments are still unique to individual sites. Rules of citizenship, practices and policies regarding accounts and passwords, the features and elementary resources of the operating system, the structuring of public directories, backup schedules, after-hours access, licensing restrictions in force on proprietary software, and numerous other aspects are subject to considerable local variation. The emerging book will make no attempt to constrain local options in these matters. Throughout the book, individual users are directed to a publication called the Local Guide for site-specific particulars. A suggested template for that guide will be provided, but it will require editing to reflect local practices.

The desired flexibility to tailor the book to a variety of circumstances would be unattainable without $\mathrm{H}_{\mathrm{E}} \mathrm{X}$. Of particular significance, $\mathrm{L}_{\mathrm{T}} \mathrm{TX}$ is able to decide in response to conditional statements controlled by Boolean flags which files should be included and which omitted in any particular processing run. The procedure has already passed its proof-of-concept trial, and the publisher 
Table 5. Sections in the chapter on integration.

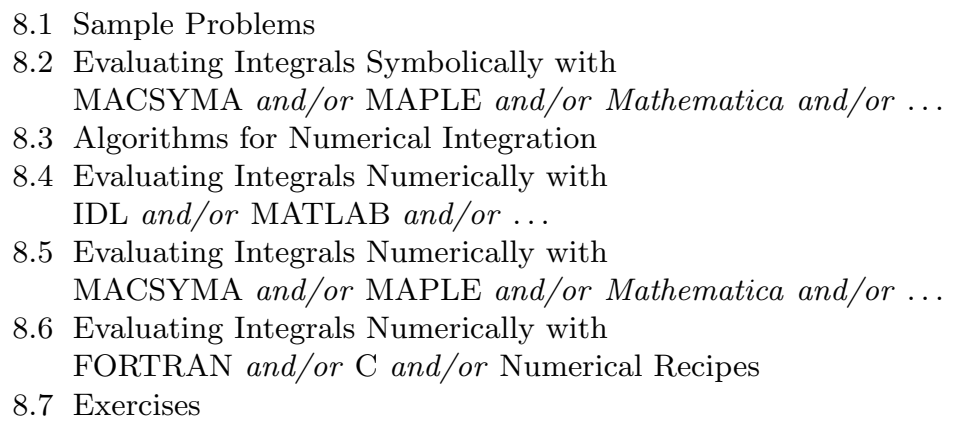

Table 6. Sample problems in Section 8.1.

\author{
8.1.1. One-Dimensional Trajectories \\ 8.1.2. Center of Mass \\ 8.1.3. Moment of Inertia \\ 8.1.4. Large-Amplitude Pendulum (Elliptic Integrals) \\ 8.1.5. The Error Function \\ 8.1.6. The Cornu Spiral \\ 8.1.7. Electric/Magnetic Fields and Potentials \\ 8.1.8. Quantum Probabilities
}

Brooks-Cole is committed to refining this essential procedure so as to be able to make a commercially feasible product. Further, once the structure has been fully worked out, contributiions from other authors may be added, so - over time - the product could expand to accommodate a wider and wider spectrum of hardware and software and include topics not originally incorporated. We sincerely hope that this book, emerging as it has from a dozen years of experience and development at Lawrence, will support efforts at other institutions to embed meaningful computational components in their undergraduate curricula.

\title{
References
}

1. NSF ILI Grant DUE-8851685 for $\$ 49433$, awarded in June, 1988 , for a project entitled "Scientific Workstations in Undergraduate Physics"; NSF ILI Grant DUE9350667 for $\$ 48777$, awarded in June, 1993, for a project entitled "Partial Differential Equations in Advanced Undergraduate Physics."

2. Keck Grant \#880969 for $\$ 200,000$, awarded in June, 1988, to support integrating scientific workstations into the physics curriculum; Keck Grant \#931348 for $\$ 250,000$, awarded in December, 1993 , to support the enhancement of advanced theoretical, computational and experimental courses. 
3. David M. Cook, "Computers in the Lawrence Physics Curriculum" Part I, Comput. Phys. 11(3; May/Jun, 1997), 240-245; Part II, Comput. Phys. 11(4; Jul/Aug, 1997), 331-335.

4. David M. Cook, "Incorporating Uses of Computational Tools in the Undergraduate Physics Curriculum" in Computing in Advanced Undergraduate Physics, the proceedings of a Sloan-supported conference held at Lawrence University, 13-14 July 1990, edited by David M. Cook and published in November, 1990, by Lawrence University.

5. David M. Cook, "Computational Exercises for the Upper-Division Undergraduate Physics Curriculum," Comput. Phys. 4(3; May/June), 308-313 (1990).

6. David M. Cook, "Introducing Computational Tools in the Upper-Division Undergraduate Physics Curriculum," Comput. Phys. 4(2; Mar/Apr), 197-201 (1990)

7. NSF CCLI-EMD Grant DUE-9952285 for $\$ 177,000$, awarded in February, 2000, for a project entitled "Strengthening Computation in Upper-Level Undergraduate Physics Programs."

8. Available from Microsoft, Seattle, Washington.

9. Available from Research Systems, Inc., Boulder, Colorado.

10. Available from The MathWorks, Inc., Natick, Massachusetts.

11. Available from Numerical Recipes Software, Cambridge, Massachusetts.

12. LSODE (the Livermore Solver for ODEs) is a component in the package ODEPACK, which is in the public domain and is available for ftp transfer from appropriate sites, e.g., www.netlib.org.

13. Available from Waterloo Software, Waterloo, Ontario.

14. Available from Wolfram Research, Inc., Champaign, Illinois.

15. Available from Synergy Software, Reading, Pennsylvania.

16. Available from Numerical Algorithms Group, Downers Grove, Illinois.

17. Available for many platforms via ftp transfer from several archives around the world. For information, start at www.tug.org.

18. Tgif is a program for drawing assorted diagrams and writing those descriptions in files of several different types. For information, go to www.ucla.edu and search for tgif.

19. Available from Vernier Software and Technology, Beaverton, Oregon.

20. SPICE is a tool for simulating the behavior of electric circuits. For information, start at www. berkeley. edu and search for SPICE.

21. Vernon D. Barger and Martin G. Olsson, Classical Mechanics: A Modern Perspective (McGraw-Hill, New York, 1995), Second Edition.

22. David J. Griffiths, Introduction to Electrodynamics (Prentice-Hall, Upper Saddle River, New Jersey, 1999), Third Edition.

23. David M. Cook, Computation and Problem Solving in Undergraduate Physics (Brooks-Cole, Pacific-Grove, CA, expected January, 2003). 